Sādhanā Vol. 37, Part 5, October 2012, pp. 609-628. (C) Indian Academy of Sciences

\title{
Cut-HDMR-based fully equivalent operational model for analysis of unreinforced masonry structures
}

\author{
D MUKHERJEE, B N RAO* and A M PRASAD
}

Structural Engineering Division, Department of Civil Engineering, Indian Institute of Technology Madras, Chennai 600 036, India

e-mail: bnrao@iitm.ac.in

MS received 8 March 2011; accepted 3 February 2012

\begin{abstract}
Mesoscale models are highly competent for understanding behaviour of unreinforced masonry structures. Their only limitation is large computational expense. Fully Equivalent Operational Model forms an equivalent mathematical model to represent a particular phenomenon where explicit relationship between inputs and outputs are unknown. This paper explores the ability of a major variant of High Dimensional Model Representation (HDMR) technique, namely Cut-HDMR, to construct the most efficient Fully Equivalent Operational Model for nonlinear finite element analysis of mesoscale model of an unreinforced masonry structure. Conclusions are reached on various aspects such as, suitability of interpolation schemes and order of Cut-HDMR approximation.
\end{abstract}

Keywords. Fully equivalent operational model; high dimensional model representation; unreinforced masonry; mesoscale modelling; moving least squares approximation.

\section{Introduction}

Unreinforced masonry (URM) is a composite material formed by periodic arrangement of unit and mortar. Around the globe, considerable proportion of residential buildings and monuments are unreinforced masonry structures. Therefore, it is of utmost importance to understand the detailed behaviour of URM. This will ensure establishment of more reliable and efficient building codes, more effective retrofitting strategies. Mesoscale modelling technique is the most suitable modelling approach which can simulate the local behaviour of URM, effectively (Zucchini \& Lourenco 2004).

In simplified micro-models or mesoscale models, masonry units are modelled with continuum elements, while mortar joints are represented by means of zero thickness interface elements (Page 1978; Lotfi \& Shing 1994; Lourenco \& Rots 1997; Giambanco et al 2001; Chaimoon \& Attard 2007). This technique allows the failure modes of a URM structure to be captured precisely. Furthermore, interpretation of analysis results is much more transparent, since the

*For correspondence 
material properties obtained from the experiment are directly used for simulation without any further simplification. However, the high computational expense involving mesoscale modelling technique, often discourages the researchers and practicing engineers. Moreover, this expense increases multiple folds when repetitive analyses are performed, such as in case of reliability studies.

A Fully Equivalent Operational Model (FEOM) (Wang et al 2005; Shorter et al 1999; Li et al 2001) is used to construct an equivalent mathematical model where relationship between input and response of an existing mathematical model is not known explicitly. FEOM is very effective when the existing model is computationally cumbersome and requires repetitive analyses. With the help of FEOM technique, required response can be obtained within fraction of time from the equivalent mathematical model.

In the present work, the potential of a major variant of high dimensional model representation (HDMR), namely Cut-HDMR, is explored for construction of FEOM. Its capabilities are illustrated with the help of numerical examples and later extended to construct FEOM for non-linear finite element analysis (FEA) of mesoscale model of a URM shear wall with an opening. Efficiency of different interpolation schemes such as Lagrange Interpolating Polynomial (LIP) and Moving Least Squares (MLS) approximation is investigated. A study is conducted on suitability of various weight functions such as, cubic spline, quartic spline and exponential weight functions for construction of MLS interpolation scheme. Recommendations are also made regarding suitability of number of sample points required to construct the response function and required order of Cut-HDMR approximation.

In this paper, a brief introduction to HDMR and its variant Cut-HDMR is given in section 2. Later, the concept of MLS interpolation scheme is discussed in section 3. In the fourth section, few mathematical examples are solved in order to demonstrate the capability of Cut-HDMR and suitability of interpolation schemes. In the fifth section, FEA of mesoscale model of URM shear wall is carried out and validated. Construction of FEOM using Cut-HDMR is carried out and given in section 6. Finally, conclusions are provided in section 7.

\section{High dimensional model representation}

Understanding of any physical phenomenon involves identifying the input variables and output response as well as mapping the input-output (IO) relationship. As the number of input variable increases, the computational complexity in mapping IO relationship increases exponentially. This is commonly termed as curse of dimensionality (Li et al 2001; Rabitz \& Alis 1999; Alis \& Rabitz 2001). HDMR (Li et al 2001; Rabitz \& Alis 1999; Alis \& Rabitz 2001; Rabitz et al 1999; Sobol 2003) drastically reduces the computation effort by expressing the response in terms of hierarchical correlated function expansions.

HDMR expansion is expressed in terms of summation of component functions. The component functions are ordered starting from a constant term to gradually approaching to multivariance as the order of approximation is increased. Let $\mathbf{x}=\left[x_{1}, x_{2}, x_{3}, \ldots, x_{N}\right]$ be $N$ dimensional vector with $N$ ranging up to $10^{2}-10^{3}$. HDMR expresses response function $f(\boldsymbol{x})$ as follows,

$$
\begin{aligned}
f(x)= & f_{0}+\sum_{i=1}^{N} f_{i}\left(x_{i}\right)+\sum_{1 \leq i \leq j \leq N} f_{i j}\left(x_{i}, x_{j}\right)+\sum_{1 \leq i \leq j \leq k \leq N} f_{i j k}\left(x_{i}, x_{j}, x_{k}\right)+\ldots \\
& +f_{12 \ldots N}\left(x_{1}, x_{2}, \ldots, x_{N}\right)
\end{aligned}
$$


where $f_{0}$ stands for the response at mean of input sample space and $f_{i}\left(x_{i}\right)$ is the first-order term expressing the response on varying $x_{i}$ independently (although nonlinearly) over the sample space. Similarly, the function $f_{i j}\left(x_{i}, x_{j}\right)$ takes into account the correlation between $x_{i}$ and $x_{j}$. The last term indicates residual contribution of $N^{t h}$ order correlated contribution of all the input variables. The basic conjecture underlying HDMR is that in real problems lower order correlations are having more influence than higher order (Li et al 2001; Alis \& Rabitz 2001).

HDMR expansions can be broadly categorized as RS (Random Sampling)-HDMR and CutHDMR. In case of RS-HDMR, the component functions are determined by integrating over randomly scattered sample points. The component functions in Cut-HDMR expansion are evaluated along lines or planes or volumes (i.e., cuts) with respect to a reference point in sample space. In this paper, only the potential of Cut-HDMR for construction of FEOM is explored.

\subsection{Cut-HDMR}

The component functions in Cut-HDMR are determined with respect to a defined reference point $\boldsymbol{c}=\left\{c_{1}, c_{2}, \cdots, c_{N}\right\}$. In the convergence limit, when all correlated functions in Eq. (1) are considered, Cut-HDMR is invariant to the choice of reference point $c$. However, in practice the choice of reference point $c$ is very important for Cut-HDMR especially if only terms up to firstand second-order in Eq. (1) are considered and it is shown that it is optimal to choose reference point $\boldsymbol{c}$ as mean values of the input variables (Sobol 2003). The component functions pose the following forms,

$$
\begin{gathered}
f_{0}=f(\boldsymbol{c}), \\
f_{i}\left(x_{i}\right)=f\left(x_{i}, \boldsymbol{c}^{i}\right)-f_{0}, \\
f_{i}\left(x_{i}, x_{j}\right)=f\left(x_{i}, x_{j}, \boldsymbol{c}^{i j}\right)-f_{i}\left(x_{i}\right)-f_{j}\left(x_{j}\right)-f_{0},
\end{gathered}
$$

and so on, where $f\left(x_{i}, \boldsymbol{c}^{i}\right)=f\left(c_{1}, c_{2}, c_{3}, \ldots, c_{i-1}, x_{i}, c_{i+1}, \ldots c_{N}\right)$ denotes that all the input variables except $x_{i}$ are at their reference point. The $f_{0}$ term is the response of the model at reference point $\boldsymbol{c}$. The higher order terms are evaluated as cuts in the input sample space through reference point. Therefore, each first order expression $f_{i}\left(x_{i}\right)$ is calculated along its variable axis through the reference point. Similarly, second order expression $f_{i}\left(x_{i}, x_{j}\right)$ is evaluated in a plane defined by set of input variables $x_{i}$ and $x_{j}$ passing through reference point $f\left(x_{i}, \boldsymbol{c}^{i}\right)$ and $f\left(x_{i}\right.$, $\left.x_{j}, c^{i j}\right)$ can be approximated as,

$$
f\left(x_{i}, c^{i}\right)=\sum_{j=1}^{n} \phi_{j}\left(x_{i}\right) f\left(c_{1}, \cdots, c_{i-1}, x_{i}^{j}, c_{i+1}, \cdots, c_{N}\right),
$$

and,

$$
\begin{aligned}
f\left(x_{i}, x_{j}, c^{i j}\right)= & \sum_{j_{1}=1}^{n} \sum_{j_{2}=1}^{n} \phi_{j_{1} j_{2}}\left(x_{i}, x_{j}\right) \\
& \times f\left(c_{1}, \cdots, c_{i-1}, x_{i}^{j_{1}}, c_{i+1}, \cdots, c_{j-1}, x_{j}^{j_{2}}, c_{j+1}, \cdots, c_{N}\right),
\end{aligned}
$$

where $n$ denotes the number of uniformly distributed sample points including reference point $c$ along the axis of the input variables. In case of first-order Cut-HDMR, the sample points are 
spaced along lines passing through reference point. In second-order Cut-HDMR, the sample points are uniformly spaced along planes passing through reference point. In Eqs. (5) and (6), $\phi_{j}\left(x_{i}\right)$ and $\varphi_{j_{1} j_{2}}\left(x_{i}, x_{j}\right)$ denote interpolation functions. The expressions for LIP interpolation functions in one-dimension or two-dimension are defined respectively as follows,

$$
\phi_{j}\left(x_{i}\right)=\frac{\left(x_{i}-x_{i}^{(1)}\right) \cdots\left(x_{i}-x_{i}^{(j-1)}\right)\left(x_{i}-x_{i}^{(j+1)}\right) \ldots\left(x_{i}-x_{i}^{(n)}\right)}{\left(x_{i}^{(j)}-x_{i}^{(1)}\right) \cdots\left(x_{i}^{(j)}-x_{i}^{(j-1)}\right)\left(x_{i}^{(j)}-x_{i}^{(j+1)}\right) \ldots\left(x_{i}^{(j)}-x_{i}^{(n)}\right)},
$$

and,

$$
\begin{aligned}
\phi_{j_{1} j_{2}}\left(x_{i}, x_{j}\right)= & {\left[\frac{\left(x_{i}-x_{i}^{(1)}\right) \cdots\left(x_{i}-x_{i}^{\left(j_{1}-1\right)}\right)\left(x_{i}-x_{i}^{\left(j_{1}+1\right)}\right) \ldots\left(x_{i}-x_{i}^{(n)}\right)}{\left(x_{i}^{\left(j_{1}\right)}-x_{i}^{(1)}\right) \cdots\left(x_{i}^{\left(j_{1}\right)}-x_{i}^{\left(j_{1}-1\right)}\right)\left(x_{i}^{\left(j_{1}\right)}-x_{i}^{\left(j_{1}+1\right)}\right) \ldots\left(x_{i}^{\left(j_{1}\right)}-x_{i}^{(n)}\right)}\right] } \\
& \times\left[\frac{\left(x_{j}-x_{j}^{(1)}\right) \cdots\left(x_{j}-x_{j}^{\left(j_{2}-1\right)}\right)\left(x_{j}-x_{j}^{\left(j_{2}+1\right)}\right) \ldots\left(x_{j}-x_{j}^{(n)}\right)}{\left(x_{j}^{\left(j_{2}\right)}-x_{j}^{(1)}\right) \cdots\left(x_{j}^{\left(j_{2}\right)}-x_{j}^{\left(j_{2}-1\right)}\right)\left(x_{j}^{\left(j_{2}\right)}-x_{j}^{\left(j_{2}+1\right)}\right) \ldots\left(x_{j}^{\left(j_{2}\right)}-x_{j}^{(n)}\right)}\right] .
\end{aligned}
$$

If $s^{\prime}$ is the dimensionality of the significant correlations, then number of required full model runs using Cut-HDMR is given by

$$
\sum_{i=0}^{s^{\prime}} \frac{N !}{(N-i) ! i !}(n-1)^{i}
$$

\subsection{Construction of FEOM using Cut-HDMR}

The response function $f(\boldsymbol{x})$ can be calculated from the following steps:

(i) Using full model runs, obtain the response at uniformly spaced sample points along the axis of the input variables in Cut-HDMR.

(ii) Compute the higher order component functions $f_{i}\left(x_{i}\right), f_{i j}\left(x_{i}, x_{j}\right)$.

(iii) Add the higher order component functions with mean response $f_{0}$ to evaluate $f(\boldsymbol{x})$.

\section{Moving least squares approximation}

MLS approximation constructs and evaluates a local polynomial fit for a given function in the entire domain. Consider a function $u(x)$ over a domain $\Omega \subseteq \mathfrak{R}^{k}$ where $k=1,2,3$ and so on. Let the domain be discretized using evenly spaced sample points. Each sample point has a corresponding response $u_{I}(x)$ associated with it. The function $u(x)$ is approximated as a polynomial $u^{h}(x)$ of order $m$ with non-constant coefficients. The order of the polynomial is considered as the order of the basis function. For example, in one-dimension $u^{h}(x)$ can be written as (Lancaster \& Salkauskas 1981; Dolbow \& Belytschko 1998),

$$
\begin{gathered}
u^{h}(x)=a_{0}(x)+a_{1}(x) x, \quad \text { (Linear basis) } \\
u^{h}(x)=a_{0}(x)+a_{1}(x) x+a_{2}(x) x^{2}, \quad \text { (Quadratic basis), }
\end{gathered}
$$


where unknown parameters $a_{i}(x)$ vary with respect to $x$. The local approximation in sub-domain is given by

$$
u_{L}^{h}(x, \bar{x})=\sum_{i=0}^{m} p_{i}(x) a_{i}(\bar{x})=\boldsymbol{p}^{T}(x) \boldsymbol{a}(\bar{x}),
$$

where $\boldsymbol{p}(x)$ is a vector of compete basis functions of order $m$,

$$
\boldsymbol{p}^{T}(x)=\left[\begin{array}{lll}
1 & x & x^{2} \cdots x^{m}
\end{array}\right]
$$

and $\boldsymbol{a}(x)$ is expressed as

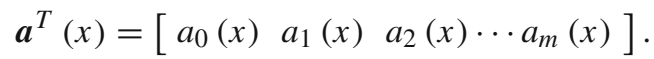

The unknown parameter $a_{i}(x)$ at any point is evaluated by minimizing the differences between the local approximation at that point and sample point responses $u_{I}(x)$. The weighted discrete $L_{2}$ norm is expressed as

$$
J=\sum_{I=1}^{m^{\prime}} w\left(x-x_{I}\right)\left[u_{L}^{h}\left(x_{I}, x\right)-u_{I}\right]^{2},
$$

where $m^{\prime}$ is the number of sample points influencing the response of the given point $x$ for which weight function $w\left(x-x_{I}\right) \neq 0$. To minimize $J$, the derivative of $J$ with respect to $\boldsymbol{a}(x)$ is set equal to zero. This lead to following set of linear equations,

$$
\boldsymbol{A}(x) \boldsymbol{a}(x)=\boldsymbol{B}(x) \boldsymbol{u},
$$

or,

$$
\boldsymbol{a}(x)=\boldsymbol{A}^{-1}(x) \boldsymbol{B}(x) \boldsymbol{u},
$$

where,

$$
\boldsymbol{A}(x)=\sum_{I=1}^{m^{\prime}} w\left(x-x_{I}\right) \boldsymbol{p}\left(x_{I}\right) \boldsymbol{p}^{T}\left(x_{I}\right),
$$

and,

$$
\begin{gathered}
\boldsymbol{B}(x)=\left[w\left(x-x_{1}\right) \boldsymbol{p}\left(x_{1}\right), w\left(x-x_{2}\right) \boldsymbol{p}\left(x_{2}\right), w\left(x-x_{3}\right) \boldsymbol{p}\left(x_{3}\right), \ldots, w\left(x-x_{m^{\prime}}\right) \boldsymbol{p}\left(x_{m^{\prime}}\right)\right], \\
\boldsymbol{u}^{T}=\left[u_{1}, u_{2}, u_{3}, \ldots, u_{m^{\prime}}\right] .
\end{gathered}
$$

Substituting back Eq. (17) in Eq. (12) the MLS interpolation scheme is obtained as

$$
u^{h}(x)=\sum_{I=1}^{m^{\prime}} \phi_{I}(x) u_{I}
$$

where shape function $\phi_{I}$ is given by

$$
\phi_{I}=\sum_{j=0}^{m} p_{j}(x)\left(\boldsymbol{A}^{-1}(x) \boldsymbol{B}(x)\right)_{j I} .
$$




\section{Analytical examples}

In this section, four numerical examples are solved to demonstrate the capability of Cut-HDMR for approximating univariate and multivariate functions. Both MLS and LIP interpolation schemes are used. A linear basis function is used for MLS interpolation scheme. The expression for exponential weight function used for MLS interpolation scheme is given by,

$$
\begin{array}{ll}
w=\frac{1}{100^{r}} & r \leq 1, \\
w=0 & r>1 .
\end{array}
$$

where $r$ is radius of influence and $w$ is weight function. In all numerical examples, to evaluate accuracy of Cut-HDMR, absolute error (AE) is calculated as,

$$
\sqrt{\frac{\left(\int_{\Omega}\left(F-F^{\prime}\right)^{2} d \Omega\right)}{\left(\int_{\Omega} F^{2} d \Omega\right)}} \times 100
$$

where $F$ is the actual response obtained from FEA and $F^{\prime}$ is the approximated response using CUT-HDMR.

\subsection{Example 1}

In this example, following piece-wise continuous function is studied,

$$
\begin{aligned}
F & =x & & 0 \leq x \leq 2, \\
& =\frac{8}{x^{2}} & & 2 \leq x \leq 4 .
\end{aligned}
$$

The function is piece-wise continuous in domain $[0,4]$ with linear variation in $[0,2]$ and quadratic variation in [2, 4]. For first-order Cut-HDMR approximation, five uniformly distributed sample points are chosen with reference point being at $x=2$. i.e., $\left\{x^{(1)}, x^{(2)}, x^{(3)}, x^{(4)}, x^{(5)}\right\}=$ $\{0,1,2,3,4\}$. The errors using LIP and MLS interpolation schemes are $21.10 \%$ and $5.43 \%$, respectively. Increasing number of sample points to seven, brings down the corresponding values to $19.35 \%$ and $2.65 \%$. Figures $1 \mathrm{a}$ and $\mathrm{b}$ show first-order Cut-HDMR approximation of the function using five and seven sample points along input variable axis, respectively.

\subsection{Example 2}

In this example, we will study the following function (Breitkopf et al 2005),

$$
F=x^{4}+12 x^{3}+21 x^{2}+12 x+20 \quad-12 \leq x \leq 4 .
$$

The function is a fourth-order polynomial in the domain $[-12,4]$. Approximation using LIP interpolation scheme matches exactly with actual function. This is quite obvious, since LIP interpolation schemes are fourth-order and sixth-order polynomial for five and seven sample points, respectively. MLS interpolation scheme shows $49.13 \%$ error using five sample points. The error 

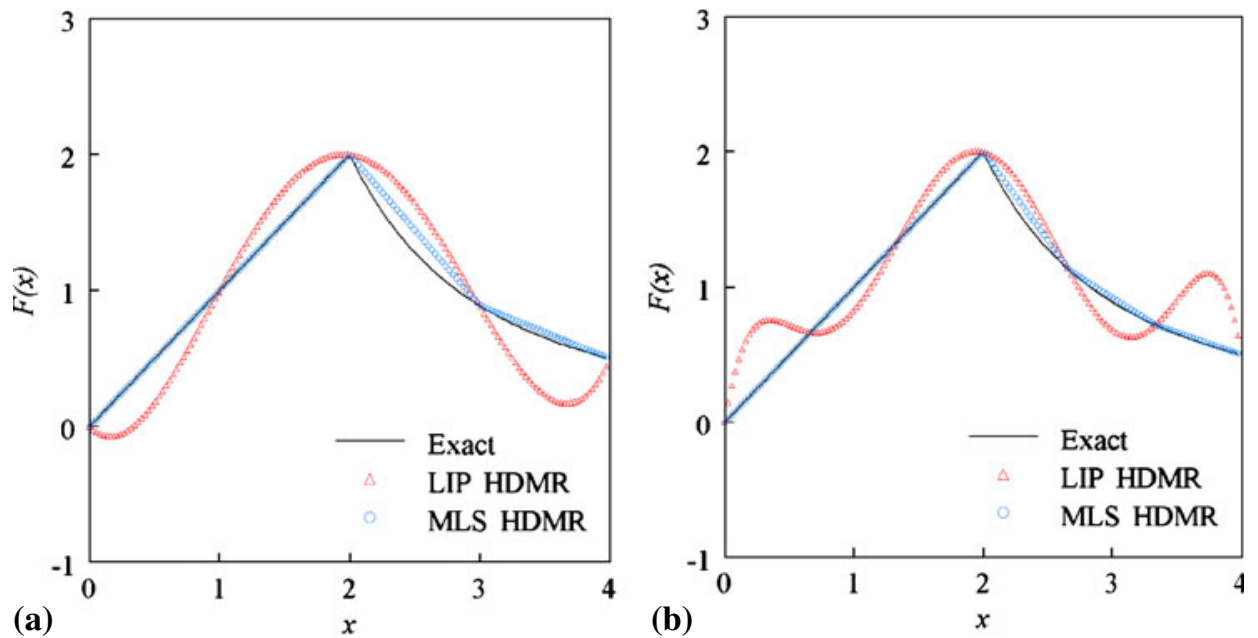

Figure 1. Plots of original function and first-order Cut-HDMR approximation using LIP and MLS interpolation scheme (Example 1): (a) $n=5 ;$ (b) $n=7$.

decreases to $26.13 \%$ after increasing the sample points to seven. Figures $2 \mathrm{a}$ and $\mathrm{b}$ show firstorder Cut-HDMR approximation of the function using five and seven sample points along input variable axis, respectively.

\subsection{Example 3}

In this example, following bivariate function is studied,

$$
\begin{aligned}
F= & 0.75 e^{\left(\frac{-(9 x-2)^{2}-(9 y-2)^{2}}{4}\right)}+0.75 e^{\left(\frac{(9 x-2)^{2}}{49}-\frac{(9 y-2)^{2}}{10}\right)}+0.50 e^{\left(\frac{-(9 x-7)^{2}-(9 y-3)^{2}}{4}\right)} \\
& -0.25 e^{\left(-(9 x-4)^{2}-(9 y-7)^{2}\right)} .
\end{aligned}
$$
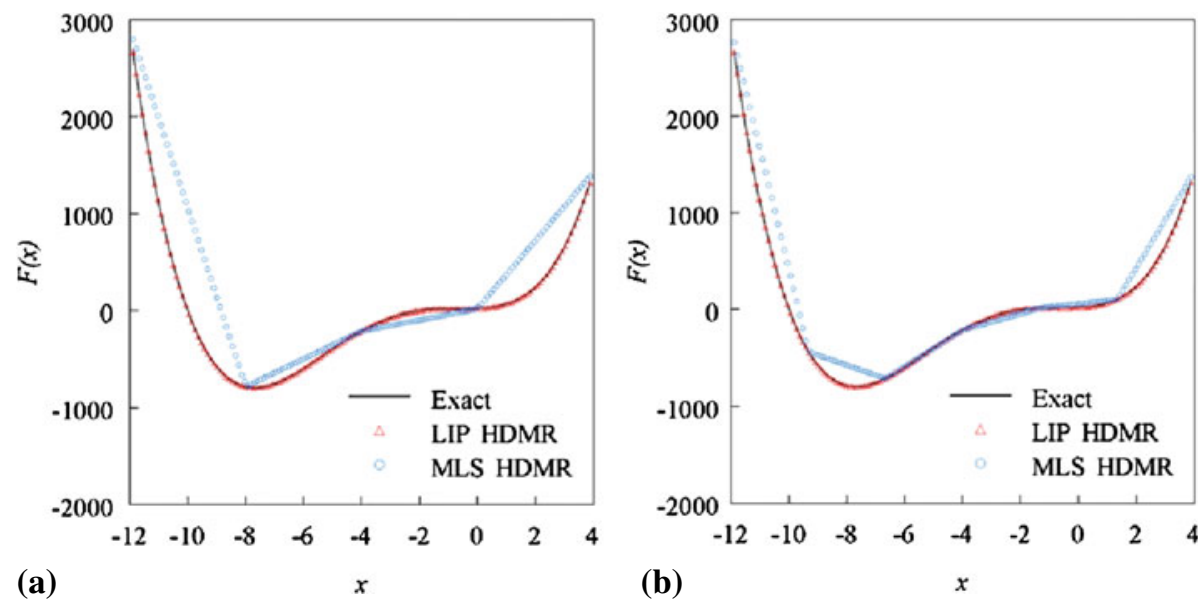

Figure 2. Plots of original function and first-order Cut-HDMR approximation using LIP and MLS interpolation scheme (Example 2): (a) $n=5$; (b) $n=7$. 


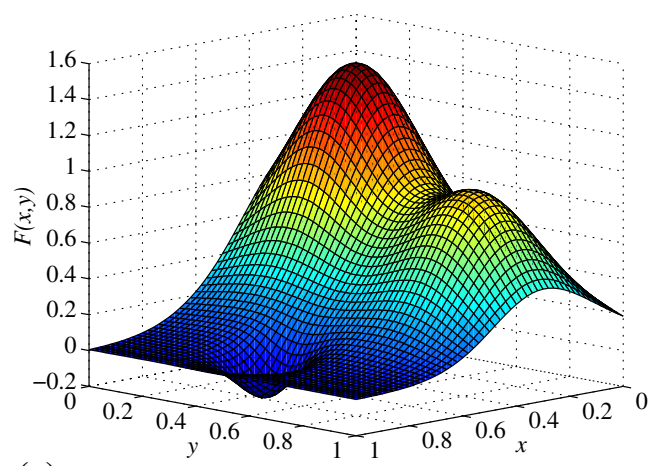

(a)

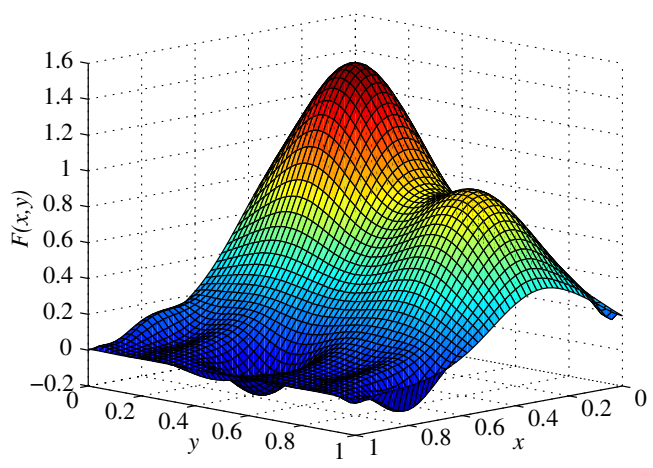

(c)

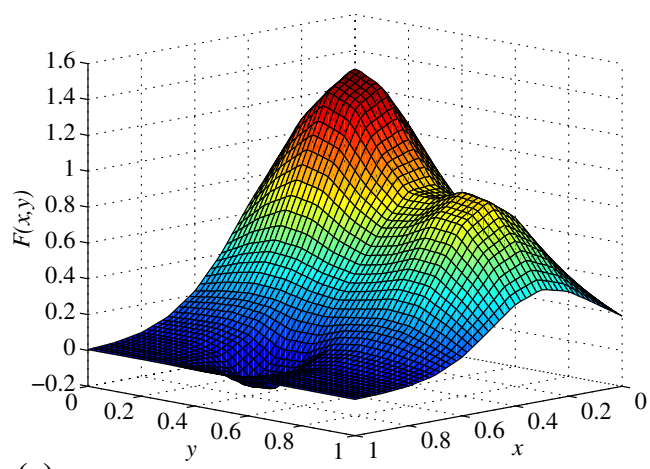

(e)
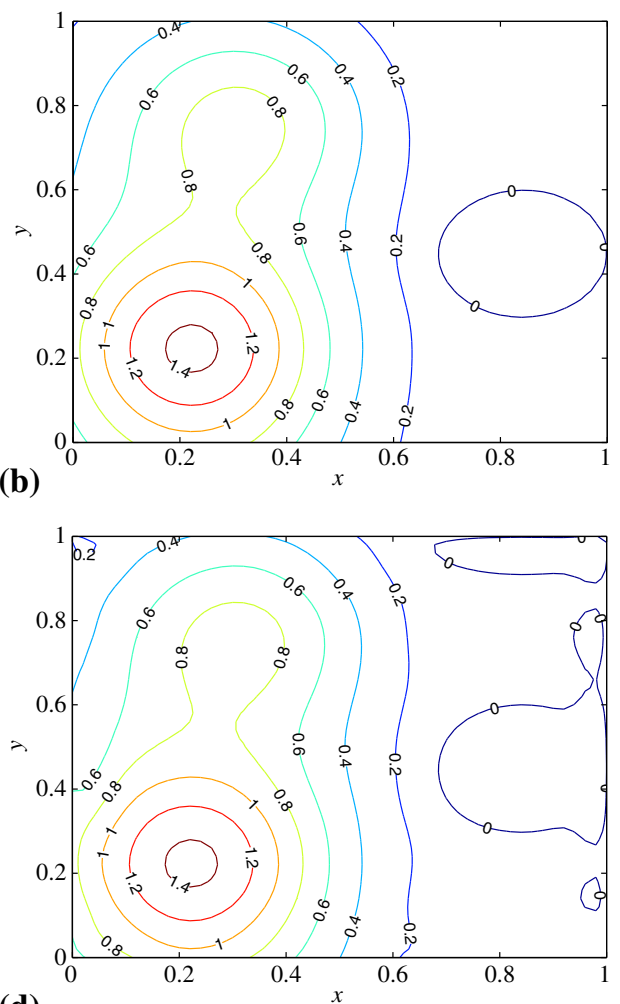

(d)

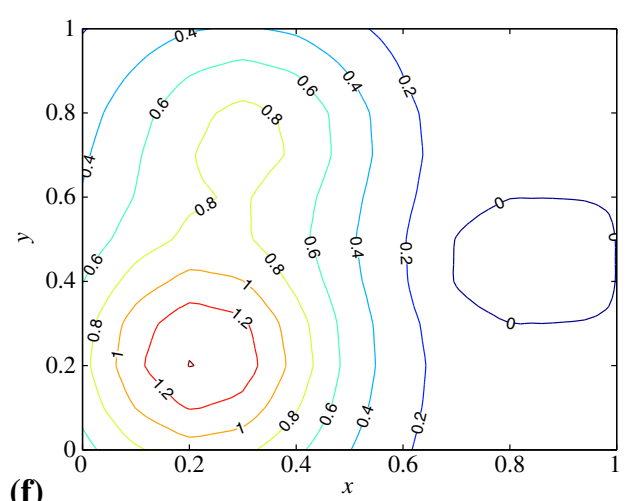

Figure 3. Approximation of Franke function using Cut-HDMR $(n=11)$. (a) Franke function; (b) Contour of Franke function; (c) LIP approximation; (d) Contour of LIP approximation; (e) MLS approximation; (f) Contour of MLS approximation.

This function is a modified form of Franke's Function which is frequently used for interpolation of scattered data. The domain of input data is defined by unit square i.e., $0 \leq x \leq 1$ and $0 \leq y \leq$ 1. The function is bimodal in nature and continuous over the domain. Approximations are made using second-order Cut-HDMR. Numbers of sample points along each input variable axis taken are 7, 9 and 11. Rectangular grids are formed within the input domain. Numbers of full function 

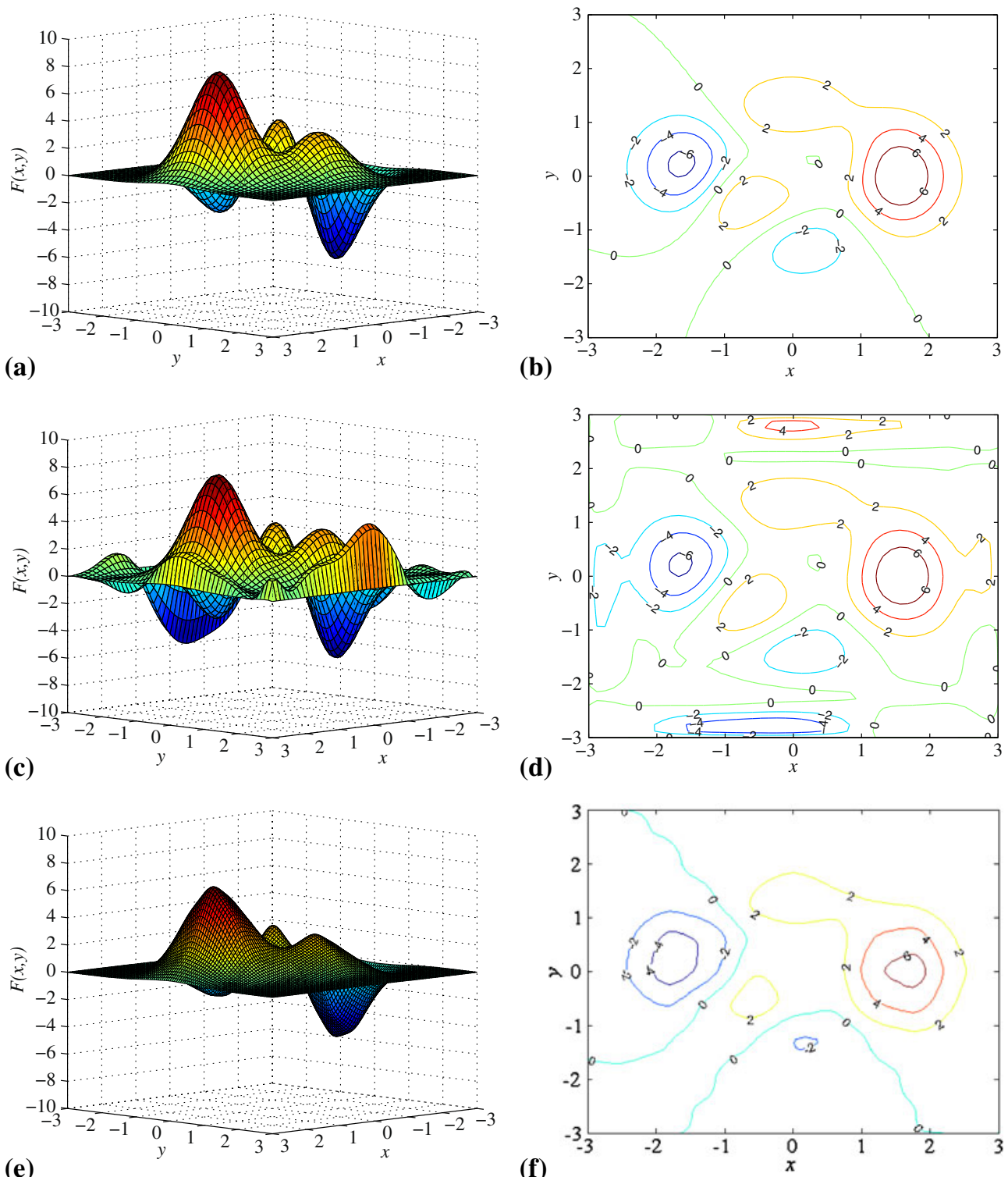

(e)

(f)

Figure 4. Approximation of Peak function using Cut-HDMR $(n=11)$. (a) Peak function; (b) Contour of Peak function; (c) LIP approximation; (d) Contour of LIP approximation; (e) MLS approximation; (f) Contour of MLS approximation.

runs are 49, 81 and 121 with increasing number of sample points. The magnitude of error using LIP interpolation scheme is 5.94\%, 5.09\% and 3.10\%, respectively. The error accumulated using MLS interpolation scheme is $7.84 \%, 4.59 \%$ and $3.00 \%$, respectively. There is no drastic variation in accuracy while changing interpolation scheme. However, MLS interpolation tends to show slightly better performance compared to LIP interpolation, as number of sample points increase. 
The error accumulated is due to the bimodal nature of the function. The plots and contours of actual function and approximated functions are shown in figures $3 \mathrm{a}-\mathrm{f}$.

\subsection{Example 4}

In this example, a two-dimensional function of the following form is considered

$$
F=3(1-x)^{2} e^{\left(-x^{2}-(y+1)^{2}\right)}-10\left(\frac{x}{5}-x^{3}-y^{5}\right) e^{\left(-x^{2}-y^{2}\right)}-\frac{1}{3} e^{\left(-(x+1)^{2}-y^{2}\right)} .
$$

The function is popularly known as Peak Function and shows multi-modal features. The input domain is defined by unit square i.e., $0 \leq x \leq 1$ and $0 \leq y \leq 1$. The peaks are formed both in positive as well as negative direction perpendicular to $x y$ plane. Second-order Cut-HDMR is used and numbers of sample points along each input variable axis taken are 7, 9 and 11. The magnitude of error using LIP interpolation scheme is $71.06 \%, 70.62 \%$ and $51.16 \%$, respectively. The error using MLS interpolation scheme is $47.86 \%, 25.05 \%$ and $18.34 \%$, respectively. In this example, MLS interpolation scheme shows significant improvement in approximation capability over LIP interpolation scheme. The accuracy of Cut-HDMR using MLS interpolation scheme is expected to rise on further increment in number of sample points. The plots and contours of actual function and approximated functions are shown in figures $4 \mathrm{a}-\mathrm{f}$.

\section{Mesoscale modelling of URM shear wall}

The test results obtained from experiments carried out in Eindhoven University of technology, Netherlands (Vermeltfoort et al 1993), are used in this work to validate the mesoscale model of a URM shear wall with an opening. Figure 5 depicts the experimental set-up. The details of the experiment are obtained from literature (Vermeltfoort et al 1993; Van Zij1 2004).

Multi-purpose finite element package DIANA (TNO DIANA BV., 2008) is used for the mesoscale modelling and numerical analysis of unreinforced masonry. Unit mortar joints and potential cracks are meshed using six-node two-dimensional zero-thickness interface elements (CL12I) with quadratic interpolation as shown in figure 6a. Four-point Newton-Cotes integration scheme is used. Steel beam and unit are modelled using eight-node quadrilateral continuum plane stress elements (CQ16M) as shown in figure 6b. Gauss integration scheme is used for construction of stiffness matrix. Figure $6 \mathrm{c}$ shows unit and interface element assemblage. MIDAS FX+ for DIANA (TNO DIANA BV., 2008) is used for generating the mesh. Mapped meshing technique is used to ensure mesh compatibility. Figure $6 \mathrm{~d}$ shows mesh discretization of interfaces around unit.

Unit is modelled as linear elastic material having a modulus of elasticity of $16.7 \mathrm{GPa}$ and having Poisson's ratio of 0.15 . Steel is considered as linear elastic material having modulus of elasticity of $200 \mathrm{GPa}$ and Poisson's ratio of 0.3 . The joint between unit and mortar is modelled using multi-surface interface model (Lourenco \& Rots 1997). Multi-surface interface model combines a tension cut-off criteria and coulomb friction criteria with compressive cap yield surface as shown in figure 7 . The normal and tangential stiffness of the interfaces can be determined using the following formulation proposed by Lourenco (1996),

$$
k_{n}=\frac{E_{u} E_{m}}{h_{m}\left(E_{u}-E_{m}\right)},
$$




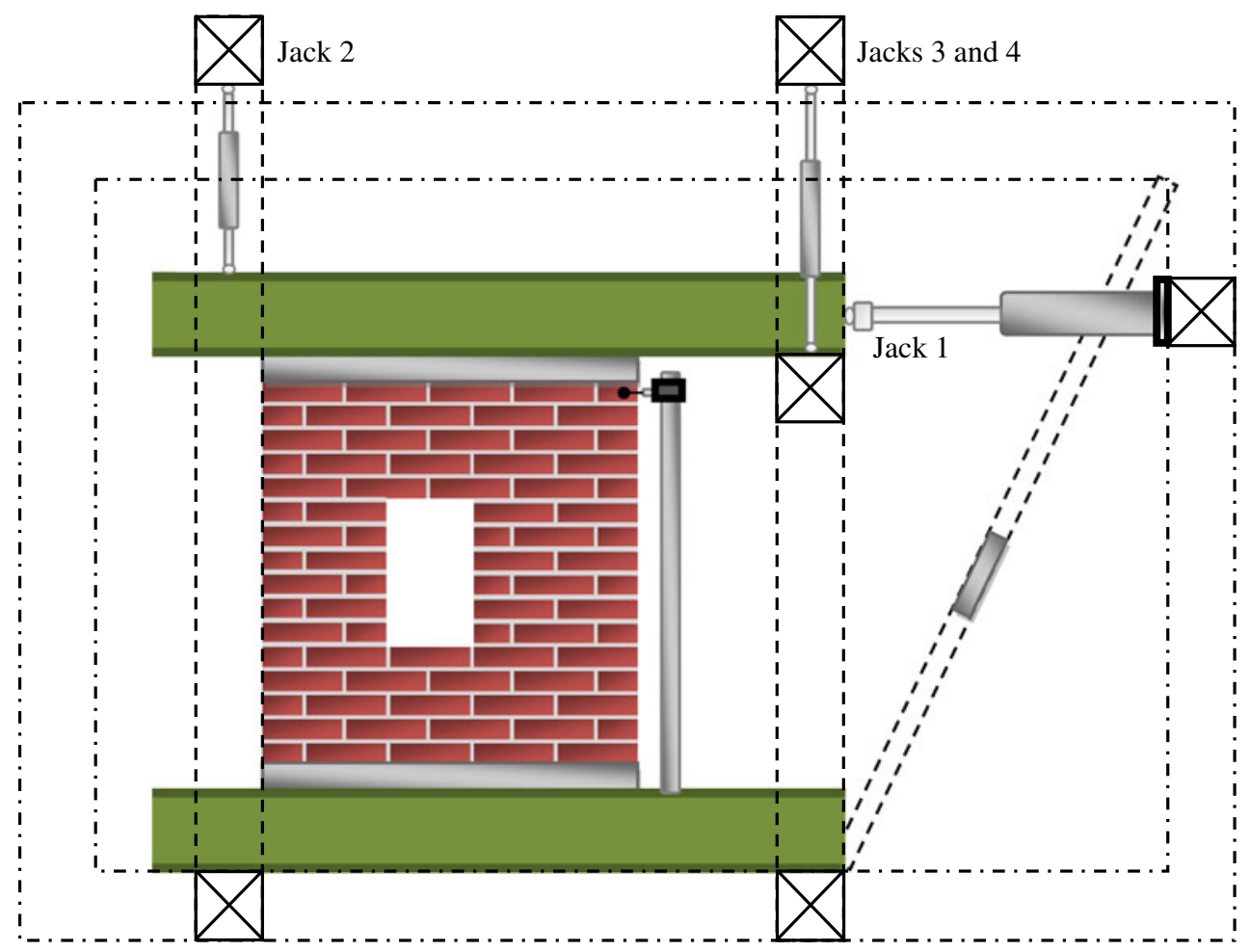

Elevation

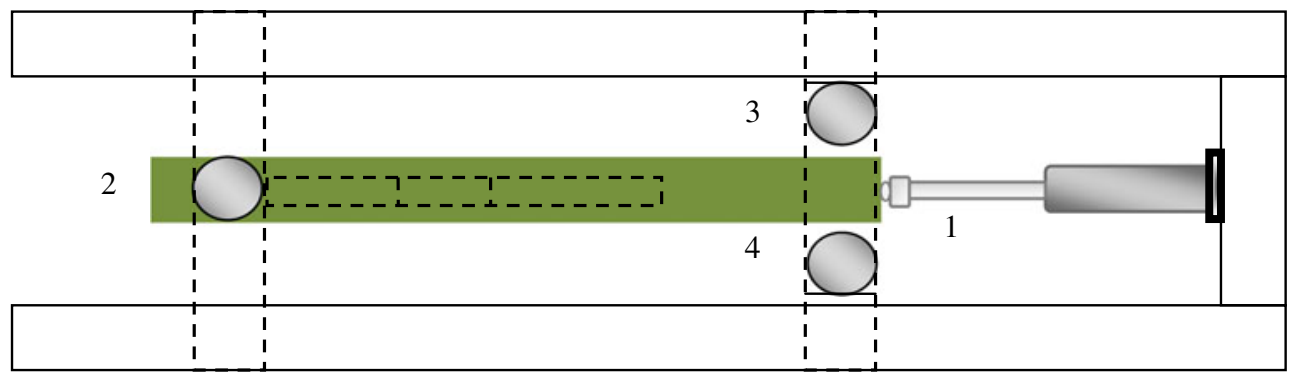

Figure 5. Details of experimental set-up (Vermeltfoort et al 1993).

$$
k_{t}=\frac{G_{u} G_{m}}{h_{m}\left(G_{u}-G_{m}\right)},
$$

where $k_{n}$ is normal stiffness of interface, $k_{t}$ is tangential stiffness of interface, $E_{u}$ is modulus of elasticity of unit, $E_{m}$ is modulus of elasticity of mortar, $G_{u}$ is modulus of rigidity of unit, $G_{m}$ is modulus of rigidity of mortar, and $h_{m}$ is thickness of mortar joint. The values of the material properties are obtained from literature (Lourenco 1996; Van Zijl et al 2001). The inelastic parameters considered in the numerical analysis are listed in table 1. Potential crack located in the middle of unit is modelled using interface elements having exponential tension softening. 

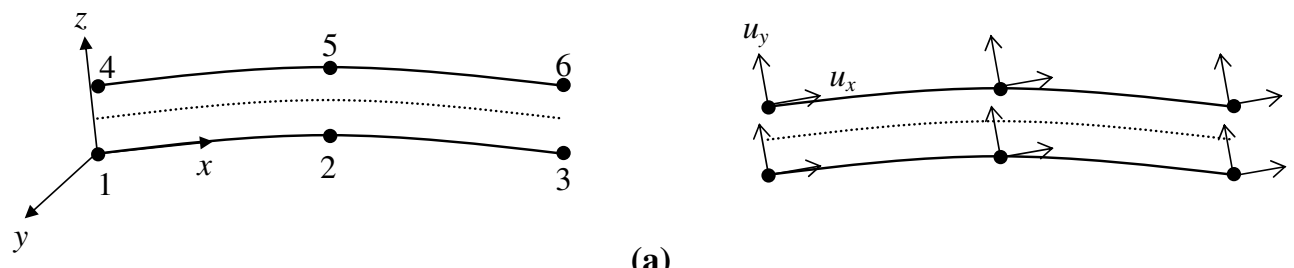

(a)

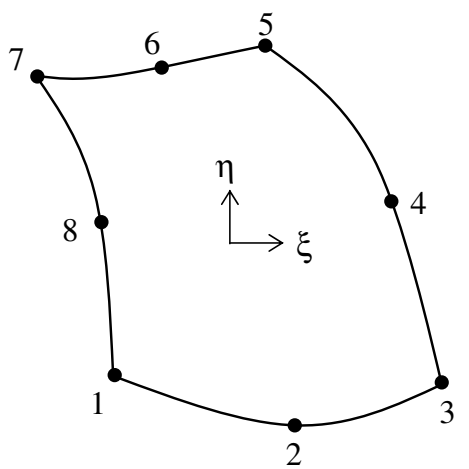

(b)

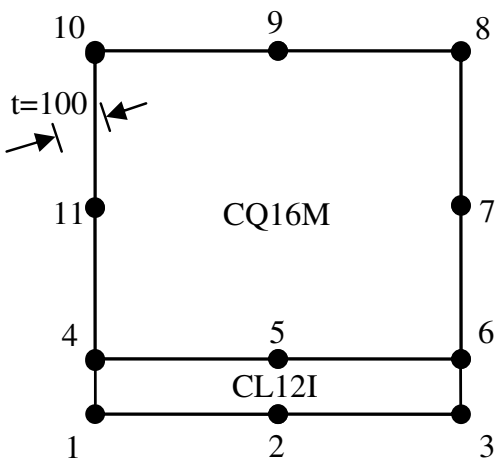

(c)
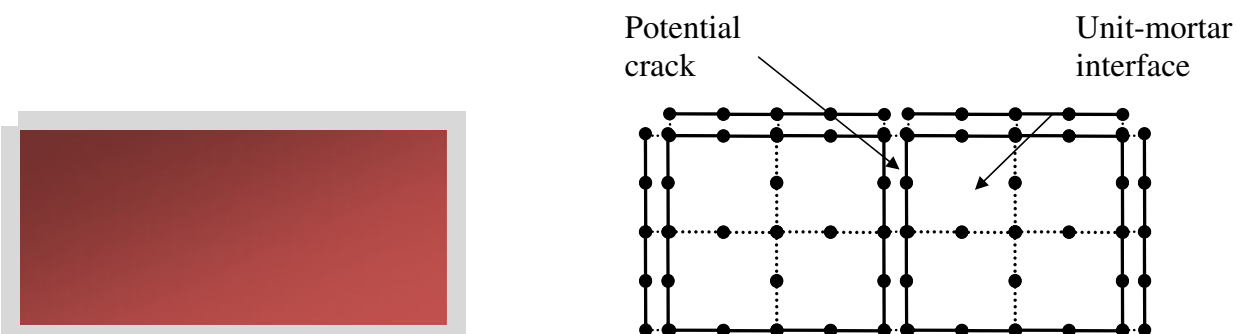

(d)

Figure 6. Details of elements. (a) Six-node two-dimensional zero-thickness interface elements; (b) Eightnode quadrilateral continuum plane stress elements; (c) Unit and interface element assemblage; and (d) mesh discretization of interfaces around unit. 


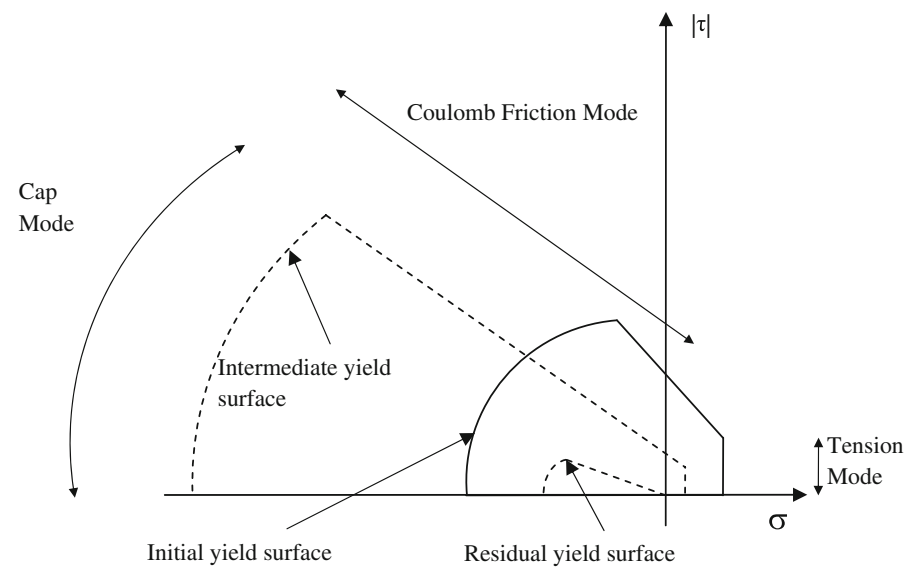

Figure 7. Multi-surface interface model (Lourenco \& Rots 1997).

Table 2 gives the details of the inelastic properties of potential cracks with exponential tension softening.

Each analysis takes approximately $12 \mathrm{~h}$ on a HP xw6600 Workstation with Quad-core Intel Xeon processor and 8 GB RAM. Figure 8 compares the load versus displacement variation at the top edge, with experimental results reported by Van Zijl et al (2001). The ductile behaviour of wall is evident from the load versus displacement curve. The sudden drops in the curve represent the development of cracks or plastic behaviour in unit. The deformed shape of the wall is shown in figure 9.

Table 1. Material properties of brick-mortar joint interface.

\begin{tabular}{lll}
\hline S1. No. & Material property & Value \\
\hline & Stiffness & \\
1 & Normal stiffness, $k_{n}\left(\mathrm{~N} / \mathrm{mm}^{3}\right)$ & 83.0 \\
2 & Tangential stiffness, $k_{t}\left(\mathrm{~N} / \mathrm{mm}^{3}\right)$ & 36.0 \\
& Tensile criterion & 0.25 \\
3 & Tensile strength, $f_{t}\left(\mathrm{~N} / \mathrm{mm}^{2}\right)$ & 0.018 \\
4 & Fracture energy in mode-I, $G_{f}^{I}(\mathrm{~N} / \mathrm{mm})$ & \\
& Shear criterion & 0.35 \\
5 & Cohesion, $C\left(\mathrm{~N} / \mathrm{mm}^{2}\right)$ & 0.75 \\
6 & Friction coefficient, $\phi$ & 0.60 \\
7 & Dilatancy coefficient, $\psi$ & 1.3 \\
8 & Confining normal stress, $\sigma_{u}\left(\mathrm{~N} / \mathrm{mm}^{2}\right)$ & 5.0 \\
9 & Shear slip degradation coefficient, $\delta$ & 0.05 \\
10 & Fracture energy in mode-II, $G_{f}^{I I}(\mathrm{~N} / \mathrm{mm})$ & 8.5 \\
& Compressive criterion & 9.0 \\
11 & Compressive strength, $f_{c}\left(\mathrm{~N} / \mathrm{mm}^{2}\right)$ & 0.093 \\
12 & Shear traction contribution factor, $C_{S S}$ & 5.0 \\
13 & Equivalent plastic relative displacement corresponding to $f_{c}, \kappa_{p}$ & \\
14 & Compressive fracture energy, $G_{f}^{c}(\mathrm{~N} / \mathrm{mm})$ & \\
\hline
\end{tabular}


Table 2. Material properties of potential crack.

\begin{tabular}{llc}
\hline Sl. No. & Material property & Value \\
\hline & Stiffness & \\
1 & Normal stiffness, $k_{n}\left(\mathrm{~N} / \mathrm{mm}^{3}\right)$ & $1.0 \times 10^{6}$ \\
2 & Tangential stiffness, $k_{t}\left(\mathrm{~N} / \mathrm{mm}^{3}\right)$ & $1.0 \times 10^{6}$ \\
& Tensile criterion & \\
3 & Tensile strength, $f_{t}\left(\mathrm{~N} / \mathrm{mm}^{2}\right)$ & 2.0 \\
4 & Fracture energy in mode-I, $G_{f}^{I}(\mathrm{~N} / \mathrm{mm})$ & 0.08 \\
\hline
\end{tabular}

\section{Construction of FEOM for analysis of URM shear wall}

Previous studies have shown that FEA of mesoscale model of URM structure is computationally expensive (Luciano \& Sacco 1997; Calderini \& Lagomarsino 2008). Therefore, it is of utmost importance to find an effective strategy to construct accurate FEOM with least computational effort. It has already been shown that Cut-HDMR drastically cuts down computational expenses compared to other standard techniques for multivariate function approximation, such as Monte-Carlo simulation (Chowdhury \& Rao 2009). However, there are numerous ways of constructing FEOM through Cut-HDMR. From the numerical examples, it can be seen that different interpolation schemes provide different degrees of accuracy depending upon the nature of function.

In this section, FEOM is formulated for the mesoscale model of URM shear wall, discussed in previous section. The geometrical details and boundary conditions are taken same as in the experiment. In addition, all other material properties are taken same as earlier, except that the exponential tension softening behaviour in the potential crack is not considered as it increases the computational effort 12 to 16 times.

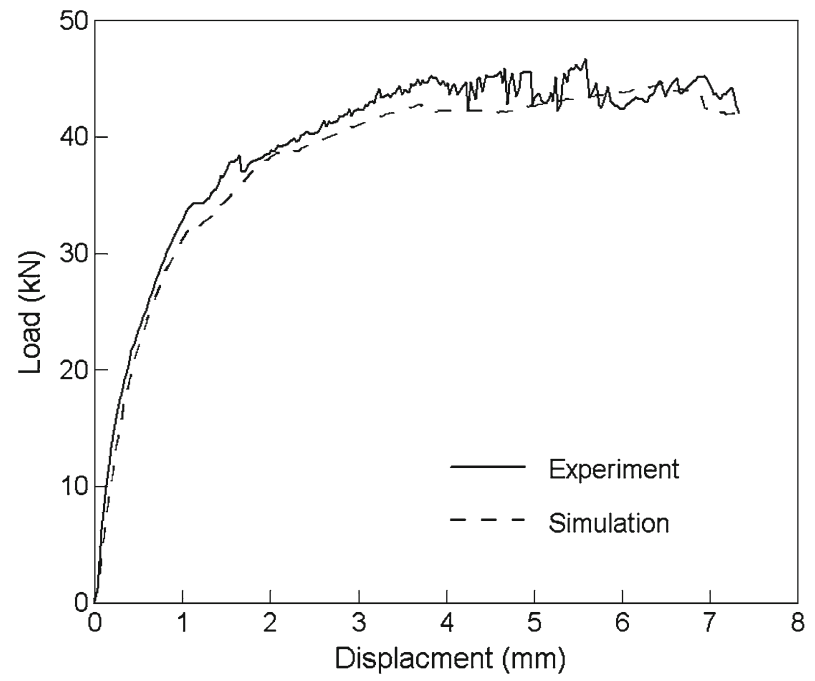

Figure 8. Comparison of load versus displacement variation at the top edge with experimental results. 


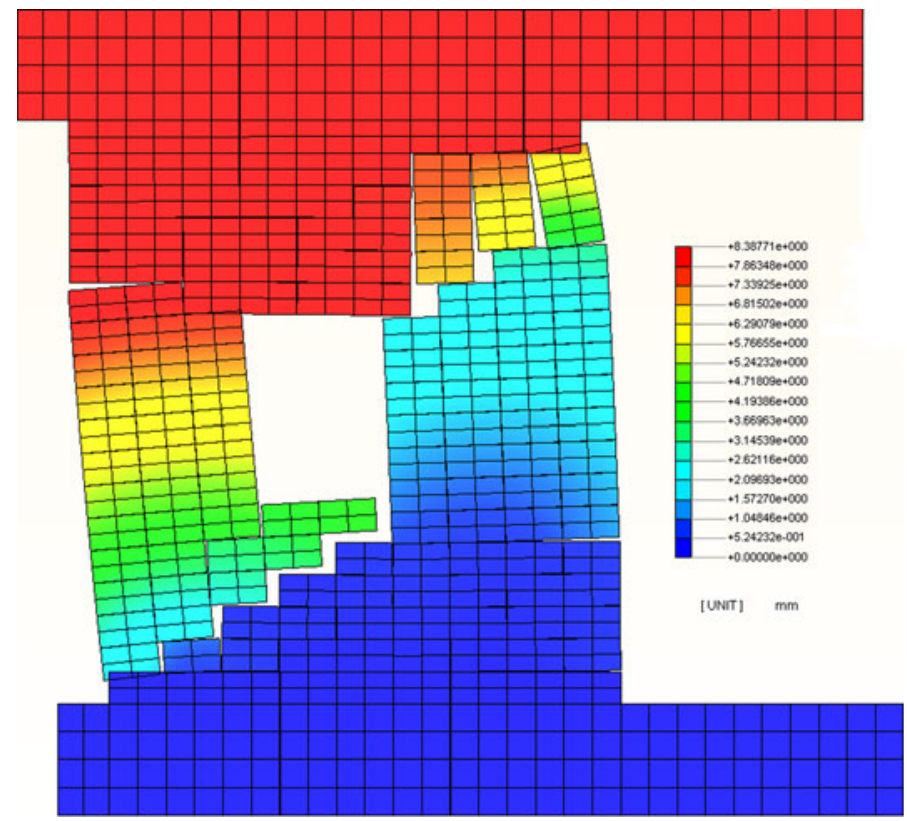

Figure 9. Deformed shape of unreinforced masonry shear wall.

\subsection{Selection and range of input variables}

For simplicity, only four input variables are taken into account. Young's modulus of mortar $\left(E_{m}\right)$ and thickness of mortar joint $\left(h_{m}\right)$ contribute to elastic stiffness of the unit-mortar interface. Compressive strength $\left(f_{c}\right)$ governs strength of the wall and compressive fracture energy $\left(G_{f}^{c}\right)$ controls the variation of compressive strength with increasing order of stress. For first three parameters, upper limit and lower limit is fixed by multiplying and dividing their original analysis values (table 1) with a factor of 0.8. A wider range (factor $=0.5$ ) is chosen for compressive fracture energy, since close estimation of this parameter is difficult. The range of parameters is decided based on two criteria: firstly, friction criteria limits tensile stress i.e., $0 \leq f_{t} \leq C / \phi$; secondly, residual cap should be greater than tension cut-off (TNO DIANA BV., 2008). It is also ensured that the mode of failure of the wall does not change within input domain. Table 3 gives the lower and upper limits of all the input parameters.

Table 3. Lower and upper limits of input parameters.

\begin{tabular}{llrr}
\hline S1. No. & Material property & Lower limit & Upper limit \\
\hline 1 & Young's modulus of mortar, $E_{m}\left(\mathrm{~N} / \mathrm{mm}^{2}\right)$ & 632.56 & 988.38 \\
2 & Joint thickness, $h_{m}(\mathrm{~mm})$ & 8.00 & 12.50 \\
3 & Compressive strength, $f_{c}\left(\mathrm{~N} / \mathrm{mm}^{2}\right)$ & 6.80 & 10.63 \\
4 & Compressive fracture energy, $G_{f}^{c}(\mathrm{~N} / \mathrm{mm})$ & 2.50 & 10.00 \\
\hline
\end{tabular}


Table 4. AE (\%) of FEOM using LIP interpolation scheme.

\begin{tabular}{lccc}
\hline Response & $n=3$ & $n=5$ & $n=7$ \\
\hline First-order approximation & & & \\
$\quad$ Initial stiffness $\left(k_{i}\right)$ & 0.6895 & 0.6704 & 0.6534 \\
$\quad$ Collapse load $\left(P_{\text {collapse }}\right)$ & 1.7366 & 2.0322 & 1.9837 \\
$\quad$ Displacement at collapse load $\left(\Delta_{\text {collapse }}\right)$ & 8.2360 & 10.1082 & 10.0114 \\
Second-order approximation & & & \\
$\quad$ Initial stiffness $\left(k_{i}\right)$ & 0.1482 & 0.0549 & 0.0591 \\
$\quad$ Collapse load $\left(P_{\text {collapse }}\right)$ & 0.9261 & 1.3456 & 0.9517 \\
$\quad$ Displacement at collapse load $\left(\Delta_{\text {collapse }}\right)$ & 6.9022 & 9.9713 & 12.5359 \\
\hline
\end{tabular}

\subsection{Methodology}

MATLAB based codes are developed for implementing algorithms to construct FEOM. For carrying out analysis, 3, 5 and 7 sample points are considered along each input variable axis. First-order approximation requires 9, 17 and 25 finite element analyses, respectively. For developing FEOM using second-order Cut-HDMR, 15, 73 and 175 analyses are performed. Both LIP and MLS interpolation schemes are implemented for evaluating component functions. An observation is also made to identify most suitable weight function for building MLS interpolation scheme. For this purpose, cubic spline and quartic spline weight functions are used along with exponential weight function defined in Eq. (23). The expressions for cubic spline and quartic spline weight functions are as follows.

Cubic spline,

$$
\begin{aligned}
& w=\frac{2}{3}-4 r^{2}+4 r^{3} \quad 0 \leq r \leq 0.5, \\
& w=\frac{4}{3}-4 r+4 r^{2}-\frac{4}{3} r^{3} \quad 0.5 \leq r \leq 1, \\
& w=0 \quad r>1 .
\end{aligned}
$$

Quartic spline,

$$
\begin{aligned}
& w=1-6 r^{2}+8 r^{3}-3 r^{4} \quad r \leq 1, \\
& w=0 \quad r>1 .
\end{aligned}
$$

Table 5. AE (\%) of FEOM using MLS approximation with exponential weight function.

\begin{tabular}{lccc}
\hline Response & $n=3$ & $n=5$ & $n=7$ \\
\hline First-order approximation & & & \\
$\quad$ Initial stiffness $\left(k_{i}\right)$ & 0.8030 & 0.6890 & 0.6673 \\
$\quad$ Collapse load $\left(P_{\text {collapse }}\right)$ & 1.8399 & 1.8934 & 1.8950 \\
$\quad$ Displacement at collapse load $\left(\Delta_{\text {collapse }}\right)$ & 8.2713 & 9.3578 & 9.3965 \\
Second-order approximation & & & \\
$\quad$ Initial stiffness $\left(k_{i}\right)$ & 0.4122 & 0.1110 & 0.0615 \\
$\quad$ Collapse load $\left(P_{\text {collapse }}\right)$ & 1.4008 & 1.0880 & 0.8862 \\
$\quad$ Displacement at collapse load $\left(\Delta_{\text {collapse }}\right)$ & 9.0037 & 8.5009 & 8.8920 \\
\hline
\end{tabular}


Table 6. AE (\%) of FEOM using MLS approximation with cubic weight function.

\begin{tabular}{lccc}
\hline Response & $n=3$ & $n=5$ & $n=7$ \\
\hline First-order approximation & & & \\
$\quad$ Initial stiffness $\left(k_{i}\right)$ & 0.8032 & 0.6890 & 0.6673 \\
$\quad$ Collapse load $\left(P_{\text {collapse }}\right)$ & 1.8398 & 1.8934 & 1.8950 \\
$\quad$ Displacement at collapse load $\left(\Delta_{\text {collapse }}\right)$ & 8.2710 & 9.3588 & 9.3965 \\
Second-order approximation & & & \\
$\quad$ Initial stiffness $\left(k_{i}\right)$ & 0.4149 & 0.1113 & 0.0615 \\
$\quad$ Collapse load $\left(P_{\text {collapse }}\right)$ & 1.4007 & 1.0873 & 0.8869 \\
$\quad$ Displacement at collapse load $\left(\Delta_{\text {collapse }}\right)$ & 9.0019 & 8.4999 & 8.8858 \\
\hline
\end{tabular}

Initial stiffness $\left(k_{i}\right)$, collapse load $\left(P_{\text {collapse }}\right)$ and displacement at collapse load $\left(\Delta_{\text {collapse }}\right)$ obtained from load-displacement curve are used as responses to be monitored. The loaddisplacement curve is measured at the location where horizontal displacement is applied. Accuracy is expressed in terms of AE, which is calculated based on responses generated by full model runs on 300 distinct set of quasi-random sample points. Sobol sequence (Sobol 1967) is used to generate the quasi-random points.

\subsection{Results and discussions}

Desired output can be obtained within 3 seconds from FEOM developed using second-order Cut-HDMR approximation with 7 sample points and MLS interpolation scheme. This implies that any response can be retrieved from the FEOM approximately 600 times faster than the corresponding mesoscale model, provided that the material and geometric parameters are defined within the input domain. Hence, a tremendous reduction in computational cost can be attained by incorporating FEOM in studies where repetitive analyses are performed.

From the results (tables 4, 5, 6 and 7), it can be noticed that second-order results are usually more accurate than first-order results ( $0.5 \%$ to $1.3 \%$ reduction in AE). Although, this higher accuracy comes at the cost of increment in number of finite element analyses required. A rise in error is observed while incrementing order of approximation, when LIP interpolation scheme is implemented to construct component functions of Cut-HDMR and displacement at collapse load is monitored as response (table 4). This occurs due to the increase in nonlinearity in

Table 7. AE (\%) of FEOM using MLS approximation with quartic weight function.

\begin{tabular}{lccc}
\hline Response & $n=3$ & $n=5$ & $n=7$ \\
\hline First-order approximation & & & \\
$\quad$ Initial stiffness $\left(k_{i}\right)$ & 0.6895 & 0.6704 & 0.6534 \\
$\quad$ Collapse load $\left(P_{\text {collapse }}\right)$ & 1.7366 & 2.0322 & 1.9837 \\
$\quad$ Displacement at collapse load $\left(\Delta_{\text {collapse }}\right)$ & 8.2360 & 10.1082 & 10.0114 \\
Second-order approximation & & & \\
$\quad$ Initial stiffness $\left(k_{i}\right)$ & 0.4136 & 0.1111 & 0.0615 \\
$\quad$ Collapse load $\left(P_{\text {collapse }}\right)$ & 1.4014 & 1.0875 & 0.8863 \\
$\quad$ Displacement at collapse load $\left(\Delta_{\text {collapse }}\right)$ & 8.9991 & 8.5012 & 8.8942 \\
\hline
\end{tabular}




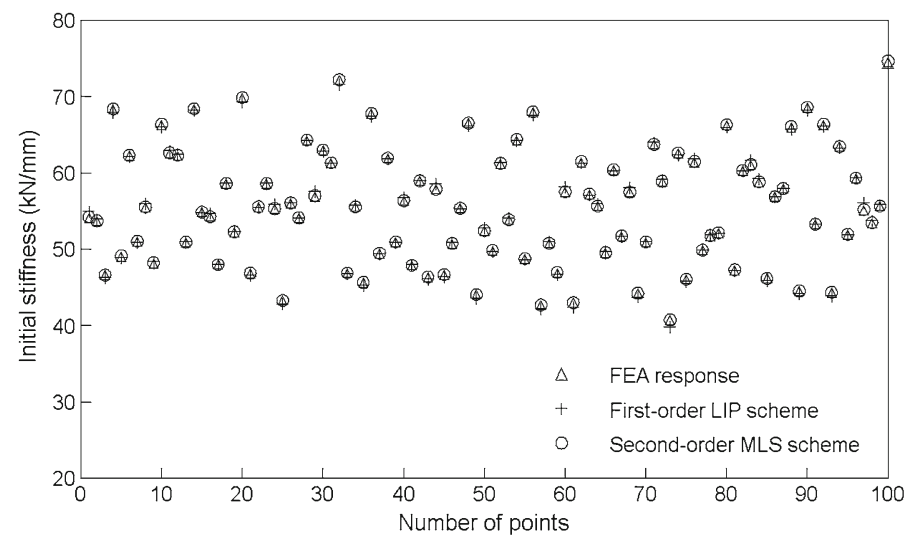

Figure 10. Comparison of sample plot for 100 quasi-random points.

response function while incrementing order of approximation. The magnitude of error is least when initial stiffness $\left(k_{i}\right)$ is monitored as response. It is followed by collapse load $\left(P_{\text {collapse }}\right)$ and displacement at collapse load ( $\left.\Delta_{\text {collapse }}\right)$.

Incrementing number of sample points $(n)$ along each input variable axis increases nonlinearity of the Cut-HDMR approximation when LIP interpolation scheme is used and therefore, does not guarantee more accurate FEOM. Since MLS interpolation scheme localizes approximation, more efficient FEOM can be formulated. One of the limitations of MLS interpolation scheme is that it does not satisfy Kronecker-delta property. This leads to a localization error when very few sample points are used to create component functions using MLS interpolation scheme. However, this error diminishes while incrementing the number of sample points $(n)$ along each input variable axis. The exponential weight function exhibits better performance as compared to other weight functions (tables 5, 6 and 7), which give singularity warning while

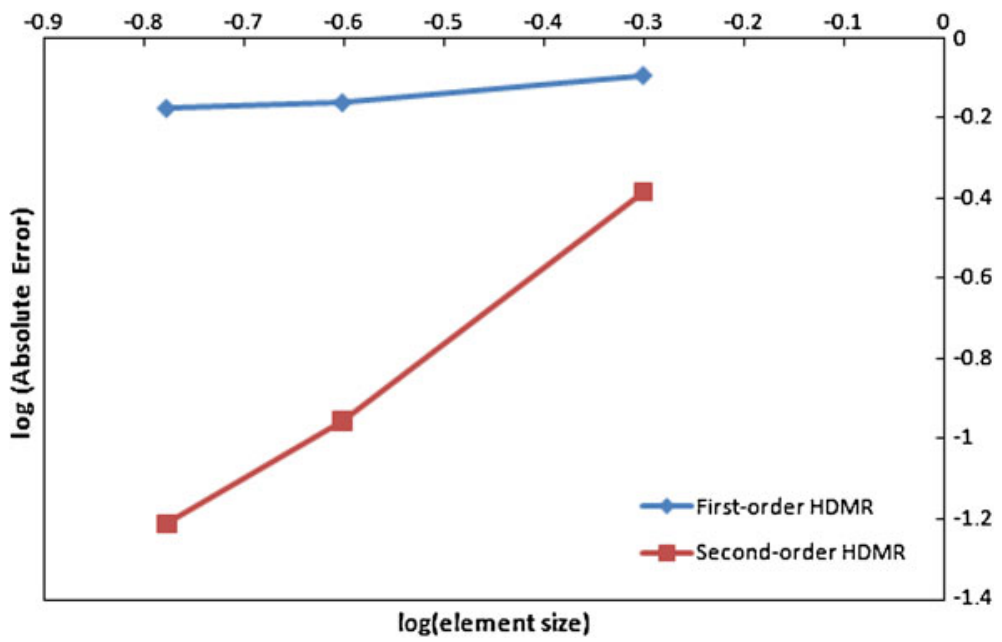

Figure 11. Convergence plot for initial stiffness in a normalized domain. 
using higher number of sample points. This is because exponential functions are $C^{\infty}$ continuous. Figure 10 shows sample plot obtained for 100 quasi-random points. In the figure, first-order Cut-HMDR constructed with LIP scheme is compared with second-order Cut-HDMR with MLS scheme. Initial stiffness $\left(k_{i}\right)$ is monitored as response. It can be observed from figure 10 that second-order Cut-HDMR constructed with MLS interpolation scheme is able to predict the FEA response more closely. Adopting this strategy restricts the magnitude of AE below 10\% for all the responses (tables 5, 6 and 7).

A further analysis is carried out in order to identify the convergence trend of FEOM constructed with first- and second-order Cut-HDMR. For this purpose, the domain of input parameters is normalized and the distance between two sample points is defined as element size. For instance, the element size for FEOM formed using 3 sample points is 0.5. Finally, a convergence plot is obtained for an elastic output parameter, initial stiffness (figure 11) using data from table 5. It can be observed from figure 11 that second-order HDMR provides better convergence than first-order. Moreover, the solution is highly convergent at initial refinement. However, further increase in number of sample points tends to reduce the convergence.

\section{Conclusions}

The present investigation gives an efficient approach to construct FEOM. Both LIP and MLS interpolation schemes are used for forming Cut-HDMR expansion. MLS interpolation scheme is constructed with linear basis function. Different weight functions are utilized for developing MLS interpolation scheme. Prior to formulate FEOM for non-linear FEA of mesoscale model of URM shear wall, four analytical functions are evaluated in order to illustrate efficiency of Cut-HDMR.

It is observed that a drastic reduction in computational expense can be achieved through FEOM when repetitive analyses of mesoscale model of URM structures are required. MLS interpolation scheme is more reliable than LIP interpolation scheme. In majority of cases, second-order approximation of Cut-HDMR shows better results compared to first-order expansion. Increasing number of sample points improves accuracy of FEOM, when MLS interpolation scheme is used. The implementation of exponential weight function for formulating MLS interpolation scheme is preferred over cubic spline and quartic spline weight functions. Considering five sample points along each input variable axis for construction of component functions is often sufficient to obtain fairly accurate FEOM. Hence, application of second-order Cut-HDMR along with MLS interpolation scheme and five sample points is recommended for most accurate FEOM. However, first-order Cut-HDMR approximation along with MLS interpolation scheme and five sample points also display reasonable accuracy and requires considerably less number of finite element analyses to form FEOM. Therefore, the preferred order of Cut-HDMR approximation should be decided solely based upon the desired degree of accuracy of FEOM.

\section{References}

Alis Ö F and Rabitz H 2001 Efficient implementation of high dimensional model representations. J. Mathemat. Chem. 29: 127-142

Breitkopf P, Naceur H, Rassineux A and Villon P 2005 Moving least squares response surface approximation: Formulation and metal forming applications. Computers \& Struct. 83: 1411-1428

Calderini C and Lagomarsino S 2008 Continuum model for in-plane anisotropic inelastic behavior of masonry. J. Struct. Eng.-ASCE 134: 209-220 
Chaimoon K and Attard M M 2007 Modeling of unreinforced masonry walls under shear and compression. Eng. Struct. 29: 2056-2068

Chowdhury R and Rao B N 2009 Assessment of high dimensional model representation techniques for reliability analysis. Probabilistic Eng. Mech. 24: 100-115

Dolbow J and Belytschko T 1998 An introduction to programming the meshless Element Free Galerkin method. Archives of Computational Methods in Engineering 5: 207-241

Giambanco G, Rizzo S and Spallino R 2001 Numerical analysis of masonry structures via interface models. Computer Methods in Applied Mechanics and Engineering 190: 6493-6511

Lancaster P and Salkauskas K 1981 Surfaces generated by moving least-squares methods. Mathematics of Computation 37: 141-158

Li G Y, Rosenthal C and Rabitz H 2001 High dimensional model representations J. Phys. Chem. A 105: $7765-7777$

Lotfi H R and Shing P B 1994 Interface model applied to fracture of masonry structures. J. Struct. Eng.ASCE 120: 63-80

Lourenco P B 1996 Computational strategies for masonry structures, Delft: Delft University

Lourenco P B and Rots J G 1997 Multisurface interface model for analysis of masonry structures. J. Eng. Mech.-ASCE 123: 660-668

Luciano R and Sacco E 1997 Homogenization technique and damage model for old masonry material. Int. J. Solids and Struct. 34: 3191-3208

Page A W 1978 Finite-element model for masonry. J. Structural Division-ASCE 104: 1267-1285

Rabitz H and Alis Ö F 1999 General foundations of high-dimensional model representations. J. Mathemat. Chem. 25: 197-233

Rabitz H, Alis Ö F, Shorter J and Shim K 1999 Efficient input-output model representations. Comput. Phys. Commun. 117: 11-20

Shorter J A, Ip P C and Rabitz H A 1999 An efficient chemical kinetics solver using high dimensional model representation. J. Phys. Chem. A 103: 7192-7198

Sobol I M 1967 On the distribution of points in a cube and the approximate evaluation of integrals. USSR Computational Mathematics and Mathematical Physics 7: 86-112

Sobol I M 2003 Theorems and examples on high dimensional model representation. Reliability Engineering \& System Safety 79: 187-193

TNO DIANA BV 2008 Diana User's Manual

Van Zijl G 2004 Modeling masonry shear-compression: Role of dilatancy highlighted. J. Eng. Mech.-ASCE 130: $1289-1296$

Van Zijl G, Rots J G and Vermeltfoort A T 2001 Modelling shear-compression in masonry, Proceedings of ninth Canadian Masonry Symposium

Vermeltfoort A T, Raijmakers T M J and Janssen H J M 1993 Shear tests on masonry walls, 6th North American Masonry Conf., 1183-1193

Wang S W, Balakrishnan S and Georgopoulos P 2005 Fast equivalent operational model of tropospheric alkane photochemistry. Aiche Journal 51: 1297-1303

Zucchini A and Lourenco P B 2004 A coupled homogenisation-damage model for masonry cracking. Computers and Struct. 82: 917-929 